\title{
Attitude of British General Practitioners towards childhood obesity in the UK
}

\author{
A. Gewargis and G. K. Grimble \\ Department of Food and Nutritional Sciences, University of Reading, RG6 6AP, UK
}

\begin{abstract}
Malnutrition and obesity are prevalent in the British Isles; occupying similar areas of social deprivation and obesity is increasing particularly among children. This is a matter of concern because of the long-term risks of CVD, cirrhosis and liver failure and stroke. The National Audit Office ${ }^{(1)}$ firmly identified the solution as lying within the remit of the Department of Health and Primary Care Trusts ${ }^{(2)}$. Obesity is now a 'medical' condition that could be tackled by General Practitioners since they interact extensively with the general public. However, in different settings across Europe and in Australia ${ }^{(3-5)}$ GP believe that this is difficult because of limited treatment efficacy, and they feel that it is a proper subject for treatment by other health-care professions. In West Berkshire, RECOMMEND (Reading Community Medical Nutrition Data) has investigated GP knowledge of and attitudes towards common forms of malnutrition.

A questionnaire was posted to $210 \mathrm{GP}$ in North Hampshire, West Berkshire and Oxfordshire. Ten questions tested nutritional knowledge (e.g. 'which of the following is a good source of folic acid?') and the remainder (19) explored medical training and clinical practice. A control group of students of Nutrition and Food Science were either in their final year of BSc $(n 26)$ or studying for an MSc $(n 8)$. Thirty GP responded (14\%). Nutritional knowledge (mean (SD), range) differed significantly between GP (5.4 (1.4), 2-7) and all students (7.1 (1.9), 3-10, $P<0.002)$, BSc students (6.7 (1.4), 3-8, $P<0.05)$ and MSc students $(8.5(1.8), 5-9, P<0.001)$. More than $95 \%$ of GP had received 'limited or no training' in any aspect of nutrition and there was no relationship between training and the test score. GP were aware of the significance of childhood obesity and $12(40 \%), 14(47 \%)$ and $5(17 \%)$ thought that it was caused by lack of physical activity, over-consumption of high energetic foods and poor parenting, respectively. GP felt ill equipped due to limited nutritional knowledge, time restraints and pressures of workload. They were sceptical about their ability to catalyse positive behavioural/dietary changes in obese children and ranked parents $(63 \%)$, teachers $(53 \%)$ and celebrities $(47 \%)$ before themselves $(23 \%)$.

These data suggest that GP in Southern Central England have little enthusiasm for tackling childhood obesity, despite their knowledge of nutrition and awareness of the importance of childhood obesity. It is unclear how this can be changed if General Practice is to meet the role envisaged in recent national reports.
\end{abstract}

1. Tackling Inequalities in Life Expectancy in Areas with the Worst Health and Deprivation (2010) The Stationery Office.

2. Nice Guidelines on Diet and CVD Prevention (2010) National Institute for Health and Clinical Excellence.

3. Brotons C, Ciurana R, Pineiro R et al. (2003) Am J Clin Nutr 77, 1048S-1051S.

4. Campbell K, Engel H, Timperio A et al. (2000) Obes Res 8, 459-466.

5. Epstein L \& Ogden J (2005) Br J Gen Pract 55, 750-754. 